\title{
Interactive comment on "Local and regional flood frequency analysis based on hierarchical Bayesian model: application to annual maximum streamflow for the Huaihe River basin” by Y. Wu et al.
}

\section{Anonymous Referee \#1}

Received and published: 27 February 2018

Summary: This study develops a hierarchical Bayesian model for regional flood frequency analysis in a region consisting of 17 annual maximum series in China. I would not consider the theoretical framework particularly novel as it is building heavily on previous work by the authors (e.g. Lima and Lall, 2010) without notable new innovations. At the same time, the comparisons with other methods is lacking somewhat in depth and rigor. In particular, I would recommend dropping the comparison with M1 as this model seems very inappropriate for the considered region. The paper does contribute to the knowledge of the flood hydrology of the considered region, but due to the before mentioned issues I do not think the manuscript contains sufficient originality and significance to be considered for publication in HESS.

Printer-friendly version

Discussion paper 
Comments:

Page 2, line 14: The most common reason for using regional frequency analysis is to obtained estimates of design events at ungauged sites.

Page 2, line 15. Sentence starting "RFA enables. .." should be deleted as it just repeats the statement made in the previous sentence.

Interactive

Page 2, lines 17-25: I think the division of regionalization methods into homogeneous regions and regression link functions unnecessarily complicated. The regression model postulates a link between, say scale-parameter and drainage area, for example $\log \left(\sigma \_\mathrm{i}\right)=\beta \_0+\beta \_1 \log \left(\mathrm{A} \_\mathrm{i}\right)$. Then a model assuming a region to be homogeneous with regards to the scale parameter would simply assume $\beta \_1=0$, so that $\log \left(\sigma \_\mathrm{i}\right.$ )$=\beta \_0$. Therefore, the first approach (homogeneous region) is simply a variation on the more general regression approach.

Page 3, line 3: Three citations to the same group for something as common as using covariates in regional flood frequency analysis? This is excessive self-citation and much more representative references should be selected here (for example some of the classical USGS reports by Benson and Thomas on this topic).

Page 3, line 5-13: The discussion of scale invariance in the existing methods is a little confusing. Stedinger and Tasker developed their GLS framework by regressing design quantiles against covariates, so presumably scale-factors are implicitly considered. Kjeldsen and Jones (2009) developed their regression framework by focusing on the median annual maximum flood, so they did not consider scale at all.

Page 3, line 21: How does an 'ordinary GEV' differ from a 'GEV' distribution? Is there an 'abnormal GEV'? I suggest using 'a GEV distribution fitted directly to the at-site data' or something like that. The phrase 'ordinary GEV' is used through-out the manuscript.

Section 2.1: I found the model description quite abstract and difficult to follow. In fact, the description of the model in section 2.2 is much more straight forward and easy to

Printer-friendly version

Discussion paper 
understand. Maybe try to incorporate the important parts from section 2.1 into a slightly more expanded version of section 2.2.

Page 4, line 12: I am not sure I understand the notation. $\varphi(\mathrm{i})=\left(\varphi^{\wedge} \mathrm{k}(\mathrm{i})\right)_{-}(\mathrm{k}=1 \ldots \mathrm{K})=\left(\varphi^{\wedge} 1\right.$ (i), .., $\left.\varphi^{\wedge} \mathrm{k}(\mathrm{i})\right)^{\wedge} \mathrm{T}$ ?

Eq. (3): In other parts of the manuscript, vectors are represented by bold characters, but here the "->" notation is adopted. I suggest being consistent and use bold notation.

Eq. (3): what is the significance of the apostrophe in D' ?

Eq. (4): This equation appears a little sudden, so maybe a bit more explanation might be useful. What does the subscript $\mathrm{H}$ signify? Is it not more common to denote a likelihood function by $L$ rather than $f$ ?

I am not sure I really understand Figure 1 . Is $K$ always equal to $L$ ?

Table 1: As you have 17 data series, what does the AIC scores refer to?

Table 1: Very unusual to see the normal distribution performing better than the lognormal distribution. Please check this is correct?

Table 1: How can the Poisson distribution be considered a candidate distribution? It is a discrete distribution, but the annual maximum series are continuous random variables? This seems inappropriate to me.

Page 5, line 15-16: $\mathrm{Y}(\mathrm{I}, \mathrm{j})$ has already been defined on page 4.

Page 5, line 21: by "a typical regional frequency model" I assume you mean the index flood method as presented by Hosking and Wallis (1997)?

Eq. (12): Not sure I understand this notation. Is this a way of writing a uniform distribution?

Page 7, line 10: Observed annual maximum flow is denoted $q$ here, but $Y$ in other parts of the manuscript?

Printer-friendly version

Discussion paper 
Page 10, line 10: Should $\mu, \beta, \sigma$ be part of the $\Lambda$ vector?

Eq. (14): I can quite comprehend if the intersite dependence between annual maximum series across sites comes into this model formulation of if this formulation assumes data from the I sites to be independent. Note that the studies by Tasker and Stedinger and Kjeldsen and Jones discussed in the introduction consider the aspects of cross-correlation in data and model errors import parts of the development of regional methods. However, these aspects are not discussed here.

Page 7, line 14: Again, a change in notation from $Y(i, j)$ to $q$ and then on to yij. Personally I prefer the latter version.

Page 8, line 4: I don't understand what is meant by 'draw values of the set of parameters' . Again, in line 10 'drawing samples'. Please explain what is meant by this?

Page 8, line 16-17: Here M1 is specified as being the index flood method based on the method of L-moments. But per comment above, I think it would be better to simply specify the model with $\beta \_0=0$ and then use the tools available to compare nested models in a likelihood framework rather than rely on the L-moments methods. Also, given the large range of drainage areas represented in the data set, I think most hydrologists would not consider this case study to qualify as a 'homogeneous region' This is also evident from the athors' figure 3, middle panel, which clearly show that the scale parameter (synonymous with L-CV) to depend on drainage area. As such, M1 is clearly inappropriate for this case. One of the main findings of the study highlighted in the abstract is that the HB-model is superior to the index flood method, but I think this is not a fair comparison.

Section 3.2: I think comparing the results for just two sites is insufficient. I would have expected at least a comprehensive cross-validation (leave-on-out) analysis at this stage.

Figure 5: Interesting to see confidence intervals that drops below zero for the at-site 
model. This is clearly inappropriate as also pointed out by the authors. Maybe use this to emphasis the value of the regional model?

Summary: There is a lot of emphasis on future work, which might or might not of course be conducted. In any case, I think there was scope for exploring some of these aspects in the current manuscript at the expense of removing the comparison with the misguided M1 model.

Interactive comment on Hydrol. Earth Syst. Sci. Discuss., https://doi.org/10.5194/hess-201822, 2018. 